\title{
The mechanical response of glassy carbon recovered from high pressure
}

\author{
X. Huang ${ }^{1 *}$, T. B. Shiell ${ }^{1}$, C. de Tomas ${ }^{2}$, I. Suarez-Martinez ${ }^{2}$, S. Wong ${ }^{3}$, S. Mann ${ }^{1}$, \\ D. R. McKenzie ${ }^{4}$, N. A. Marks², D. G. McCulloch ${ }^{3}$ and J. E. Bradby ${ }^{1}$ \\ ${ }^{1}$ Department of Electronic Materials Engineering, Research School of Physics, Australian National \\ University, Canberra, ACT 2601, Australia \\ ${ }^{2}$ Department of Physics and Astronomy, Curtin University, Perth, WA 6845, Australia \\ ${ }^{3}$ Physics, School of Science, RMIT University, Melbourne, VIC 3001, Australia \\ ${ }^{4}$ School of Physics, The University of Sydney, Sydney, NSW 2006, Australia
}

\begin{abstract}
Glassy carbon $(G C)$ is usually considered the prototypical super-elastic material which can almost fully recover its shape after compression of several gigapascals. In this work, nanoindentation is used to study the mechanical response of GC, which was subjected to a range of high pressures using a diamond anvil cell (DAC). We show that GC starts to lose its elasticity after compression to $6 \mathrm{GPa}$, and becomes clearly mechanically anisotropic after being compressed beyond $\sim 30 \mathrm{GPa}$. Molecular dynamics (MD) simulations are used to calculate the Young's modulus before and after compression. Through our experimental results and MD simulations, we show that the elasticity of GC is at a minimum around $30 \mathrm{GPa}$ but recovers after compression to higher pressures along the DAC compression axis.
\end{abstract}

*Corresponding author: xingshuo.huang@anu.edu.au 


\section{Introduction}

Glassy carbon (GC) is a predominately $s p^{2}$ bonded disordered carbon material, which is synthesized by the high temperature pyrolysis of cross-linked polymers [1,2]. Its useful properties include an ability to recover from large strains [3], termed superelasticity in the literature [4,5], and its resistance to graphitizing at high temperatures [4,6-7]. The material is also used as a pure, disordered carbon precursor for high pressure studies that enables it to form new structures without overcoming the high barriers that may be present between crystalline phases. "Amorphous diamond" and nanocrystalline hexagonal diamond have both been reported to form after compression of GC [8-13].

In a study by Hu et al. GC was compressed using a multi-anvil press at temperatures from 400 to $1000^{\circ} \mathrm{C}$ before measuring the mechanical properties of the recovered samples. This work reported changes in the mechanical properties of GC recovered from pressures up to $25 \mathrm{GPa}$ at high temperature [14]. They showed that the hardness and Young's modulus of GCs subjected to moderate pressures and temperatures were higher than those of uncompressed GC, but the elastic recovery was not significantly affected. Recent studies using Transmission Electron Microscopy (TEM) and X-ray Powder Diffraction (XRD) found that $s p^{3}$ bonds form gradually as $\mathrm{GC}$ is compressed to $\sim 30 \mathrm{GPa}$ at room temperature but these bonds revert to $s p^{2}$ hybridization after the pressure is removed [15-17]. Permanent microstructural changes are observed for pressures $\sim 40 \mathrm{GPa}[15,18,19]$. It has been proposed that the recovered GC is no longer superelastic or non-graphitizing. However, it has not been experimentally confirmed that the microstructural change of GC has effects on its mechanical properties.

Nanoindentation is used in this work to study the mechanical response of GC before and after compression. Nanoindentation of uncompressed GC shows a hysteretic load-displacement 
curve and strong elastic recovery $[3,4,20]$. This complex mechanical behaviour voids the standard analysis technique of nanoindentation curves to calculate the hardness or Young's modulus [21,22]. The Young's modulus of GC can been calculated from the stress-strain curves, which requires a spherical indentation tip and large samples that were not available in this study $[4,23]$. The size of samples probed in this study are of the order of $10^{-4} \mathrm{~mm}^{3}$, as detailed below. Thus the Young's modulus is not measured experimentally in this work, but is instead computed using molecular dynamics (MD) simulations. MD simulations are performed using the package LAMMPS [24] and the Environment Dependent Interaction Potential (EDIP) [25] are used, as in our previous work [26,27], to describe carbon-carbon interactions, where atomistic structures of GC can be generated and compressed to high pressure.

The technique adopted in this work to measure and quantify the mechanical behaviour utilizes the concept of "the indentation ductility index $(D)$ " proposed by Iwashita et al. [4], which is based on the elasto-plastic behaviour measurements of Sakai [28]. $D$ is used to calculate the elastic and plastic response of the sample, and is related to the indentation energy [29]. It is defined as the ratio of the area inside the nanoindentation hysteresis loop $\left(U_{h}\right)$ to the area under the loading curve ( $U_{\text {loading }}$ ). The elasticity index $E$ generated from the nanoindentation curve is defined as the ratio of the area under the unloading curve ( $\left.U_{\text {unloading }}\right)$ to the area under the loading curve ( $U_{\text {loading }}$ ). The definition of $E$ and its relationship with $D$ are expressed as

$$
E=\frac{U_{\text {unloading }}}{U_{\text {loading }}}=\frac{U_{\text {loading }}-U_{h}}{U_{\text {loading }}}=1-\frac{U_{h}}{U_{\text {loading }}}=1-D .
$$

According to this definition, a purely plastically-deforming sample like aluminium will yield an $E=0$, and $E=1$ is for a purely elastically-deforming sample like diamond. For comparison, fused silica, which is a commonly used reference material in nanoindentation and is considered as an elastic material [31-33], has an elasticity index of $\sim 0.74$ calculated by this approach. 


\section{Experiment}

There are various types of GC commercially available $[3,23]$. The GC analyzed in this study was purchased in the form of a solid plate from Hochtemperatur Werkstoffe (Sigradur-G). It was synthesized at above $2000^{\circ} \mathrm{C}$ and is commonly referred to as 'Type II'. A Boehler-Almaxplate DAC [34] (anvil culet diameter: $240 \mu \mathrm{m}$ ) was used to generate pressure. The stainless steel gasket was pre-indented to a thickness of $\sim 40 \mu \mathrm{m}$. A hole with a diameter of $\sim 100 \mu \mathrm{m}$ was created to act as the sample chamber. The GC plate was ground into small chips and loaded into the hole without any pressure medium, similar to our other studies $[8,15,23]$. As the pressure increased, the sample pieces were crushed to fill the chamber. A schematic of a DAC setup is shown in Fig. 1(a). In separate experiments, the samples were compressed and recovered from pressures of 6, 13, 20, 23, 30, 44 and 54 GPa. After the uniaxial compression was performed, the radius of the samples, as observed from the top, were not observed to change compared to the radius before compression. Unlike pillar compression of this material $[35,36]$ no fracture was observed via loading under these conditions.

After being subjected to pressure in the DAC, the gasket with retained sample was removed and embedded in a hard epoxy resin (EpoxySet resin, Allied High Tech Products Inc.) [Fig. 1(b)]. The pucks were mechanically polished to expose the GC sample [Fig. 1(c)]. Ted-Pella water-based $0.05 \mu \mathrm{m}$ alumina suspension was used as the final polishing slip for all samples. After the first set of nanoindentation was performed [from 'direction 1' in Fig. 1(c)], the samples were again encased in resin, cut, and polished to enable performing nanoindentation in the orthogonal direction ('direction 2'), as shown in Figs. 1(d) - (f). After polishing, the samples had a thickness ranging from $\sim 10$ to $30 \mu \mathrm{m}$. A bulk GC sample $(\sim 10 \mathrm{~mm} \times 5 \mathrm{~mm} \times 2$ $\mathrm{mm}$ ) was also indented, which was not mounted in epoxy but was polished using the same method for use as a reference sample. Finally a very low load sample was prepared by loading 
GC into a DAC and applying a pressure of less than $0.1 \mathrm{GPa}$, which was used to measure any changes in the mechanical response introduced by the mounting process.

A Renishaw micro-Raman inVia spectrometer $(532 \mathrm{~nm})$ was used to measure the pressure induced in the DAC. A ruby ball (with radius $\sim 3 \mu \mathrm{m}$ ) was loaded inside the cell and the R1ruby fluorescence line was used to determine the pressure up to 10 GPa $[37,38]$. The Raman diamond spectrum was used to determine the pressure higher than 10 GPa [39].

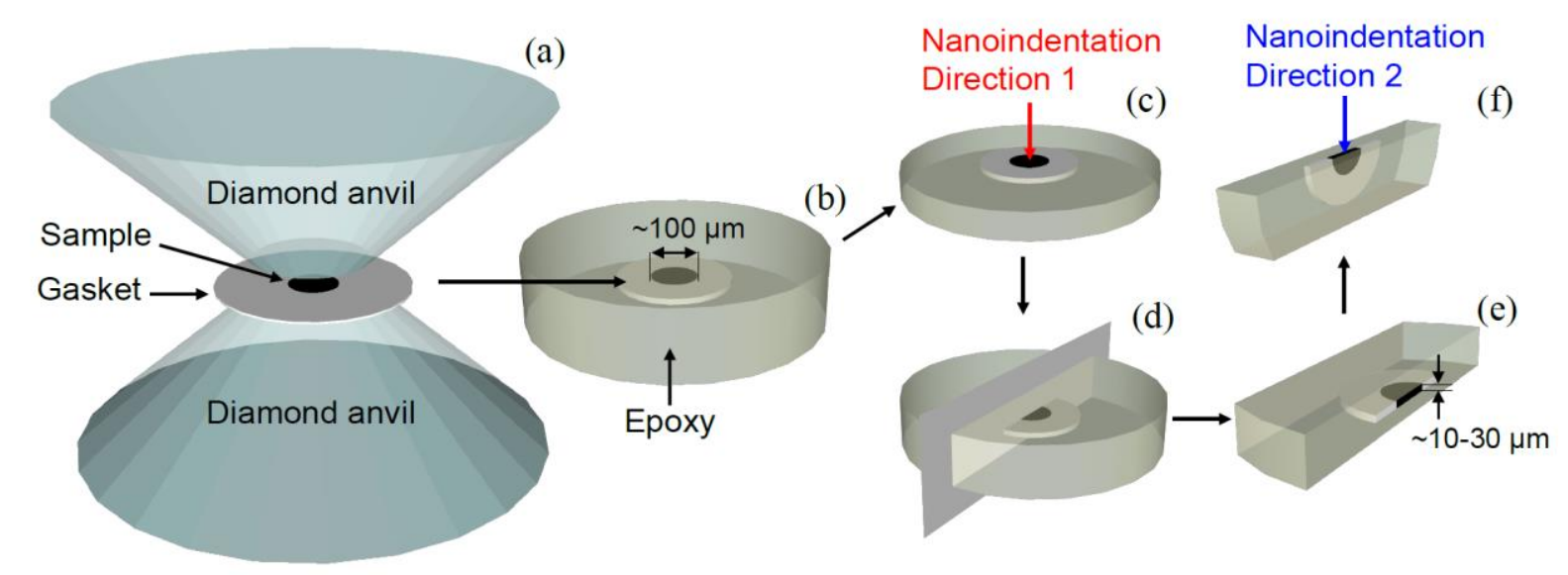

Figure 1: Schematic of the sample preparation process. (a) Glassy carbon (GC) is compressed inside a diamond anvil cell (DAC) hold by a gasket. (b) The gasket containing the sample is recovered and embedded in epoxy before (c) the sample is polished to reveal the GC (direction 1). (d) The sample is enclosed again in epoxy before being cut using a diamond saw from the side and (e) creating two parallel surfaces. (f) The sample is then polished using the same method to reveal the GC from direction 2.

The nanoindenter used in this work was a Hysitron TI 950 TriboIndenter with a Berkovich tip. Two different maximum loads were used for different purposes: $1 \mathrm{mN}$ and $12 \mathrm{mN}$, with the latter being the maximum can be applied by this system. Both loading and unloading were done 
in 10 seconds with no holding period at the maximum load. The data set created at $12 \mathrm{mN}$ was used to create a series of residual indents for imaging using the system's surface imaging capability. Using the in-built software imaging system, it was ensured that the images were collected from exactly the same indented area. The data set created at $1 \mathrm{mN}$ were used to quantify the mechanical behaviour from the load-displacement curves. This low load was used to minimize any possible influence of the epoxy substrate on numerical analysis. It should be noted that even with the higher load of $12 \mathrm{mN}$, the nanoindentation maximum depth was no more than $0.6 \mu \mathrm{m}$, which is less than $6 \%$ of the sample thickness. Thus the effect of the epoxy substrate on the mechanical response of the samples can be considered negligible $[40,41]$.

\section{Results and discussion}

Surface profile images of indented samples recovered from different pressures in the two directions are shown in Fig. 2. No significant differences were observed in the images of the residual indents between the two directions in any of the samples studied. Figure 2(a) shows very faint residual indents $\left(<10 \mathrm{~nm}\right.$ in depth $\left(\mathrm{h}_{\mathrm{r}}\right)$, obtained from the load-displacement curves) on the surface of the sample recovered after minimal loading $(<0.1 \mathrm{GPa})$. This is expected and is in keeping with previous reports that bulk GC is considered as superelastic [3]. Shallow residual indents $\left(\mathrm{h}_{\mathrm{r}}<50 \mathrm{~nm}\right)$ are observed for samples subjected to 6, 13, 20 and $23 \mathrm{GPa}$. It is surprising that residual indents are observed at these pressures, as previous studies suggested that there is only minimal change in the recovered microstructure in this pressure range, using other techniques [15]. Figure 2(c) shows that above compression of $30 \mathrm{GPa}$, clear residual indents can be observed. These indents have residual depths greater than $50 \mathrm{~nm}$.

It needs to be clarified that the exact final residual depth values could not be measured. The operation mode of the nanoindentation system is set as load control, which means the system 
will stop collecting data once the load reaches zero. However, this happens when the tip is still slightly touching the surface and there is an unknown final depth of a few nanometers. Such that the residual depths can only be separated into different ranges as defined above.

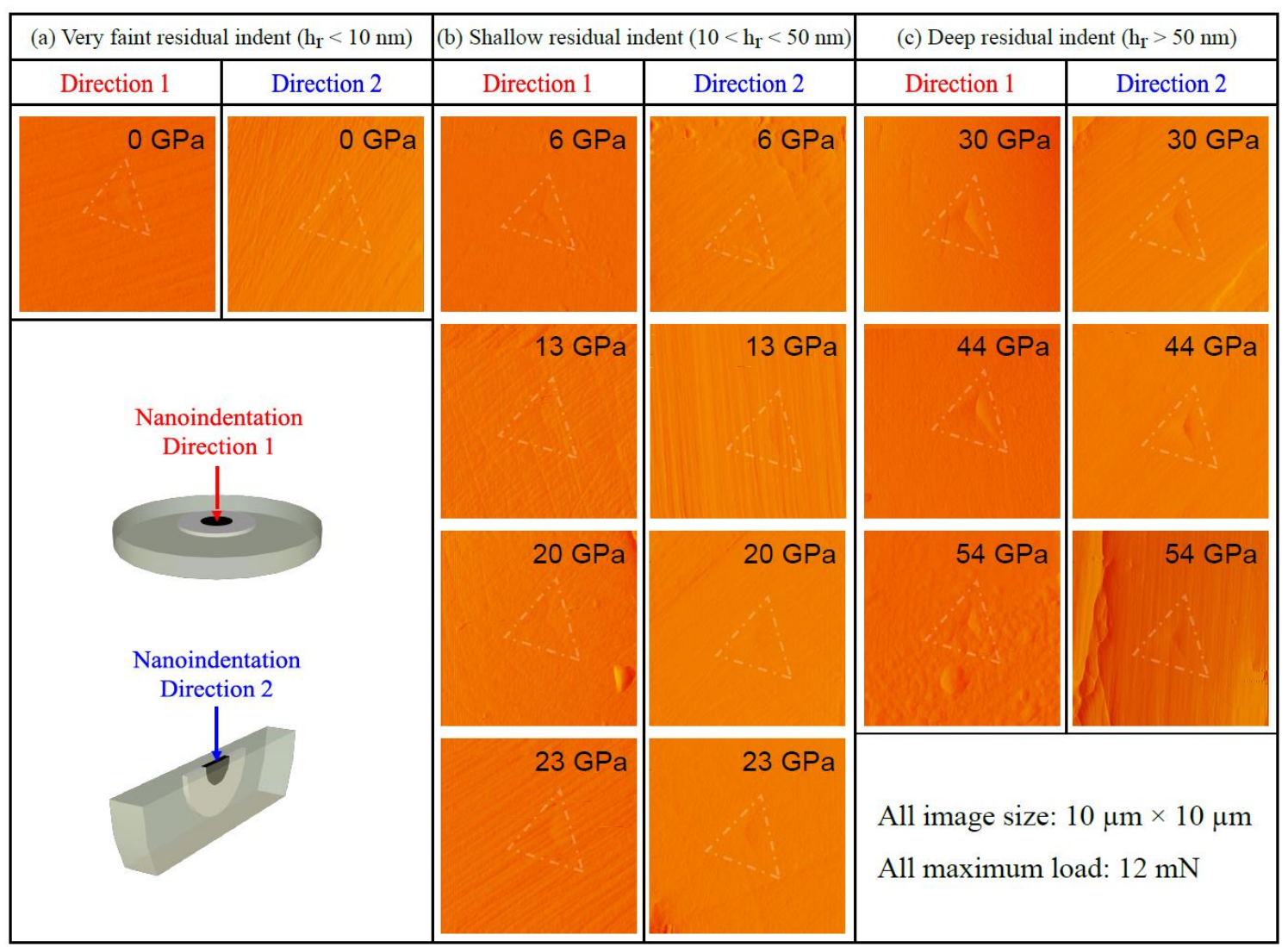

Figure 2: Scanning probe images (with size $10 \times 10 \mu \mathrm{m}$ ) of samples recovered from different pressures in both directions, parallel with and perpendicular to the DAC compression axis. The scanned images are collected immediately after nanoindentation. The indents were made using a Berkovich tip and a maximum load of $12 \mathrm{mN}$. Images are separated according to the residual depth $\left(h_{r}\right)$ from nanoindentation curves, where (a) very faint indents, (b) shallow indents and (c) clear indents are observed. 
Figure 3 shows typical nanoindentation load-displacement curves loaded to $1 \mathrm{mN}$ for GC samples subjected to pressures of 13 and $54 \mathrm{GPa}$. At maximum load of $1 \mathrm{mN}$, the tip penetrates only a maximum of $2 \%$ of the sample thickness (much less than that the samples indented to a maximum load of $12 \mathrm{mN}$ ), hence the effect of epoxy substrate can be ignored [40,41]. (A discussion on the effect of the epoxy is also given below.) In Fig. 3, solid curves are for the 13 GPa sample and dashed curves for the $54 \mathrm{GPa}$ sample. These samples were chosen as they sit below and above the $\sim 40 \mathrm{GPa}$ threshold. A curve from bulk GC is also shown for reference. The depth at the maximum load for the $13 \mathrm{GPa}$ sample is similar to that of bulk GC and also similar for both directions. This is in contrast to the 54 GPa sample, where the depth at maximum load is less in direction 2 and there is a clear difference in direction 1 and direction 2. It is noteworthy that even though the maximum depth for $54 \mathrm{GPa}$ sample shows large difference in the two directions, the residual depth has similar values. In fact, the residual depth is below $10 \mathrm{~nm}$ for all samples loaded with $1 \mathrm{mN}$ and indistinguishable within experimental uncertainty between the two directions. 


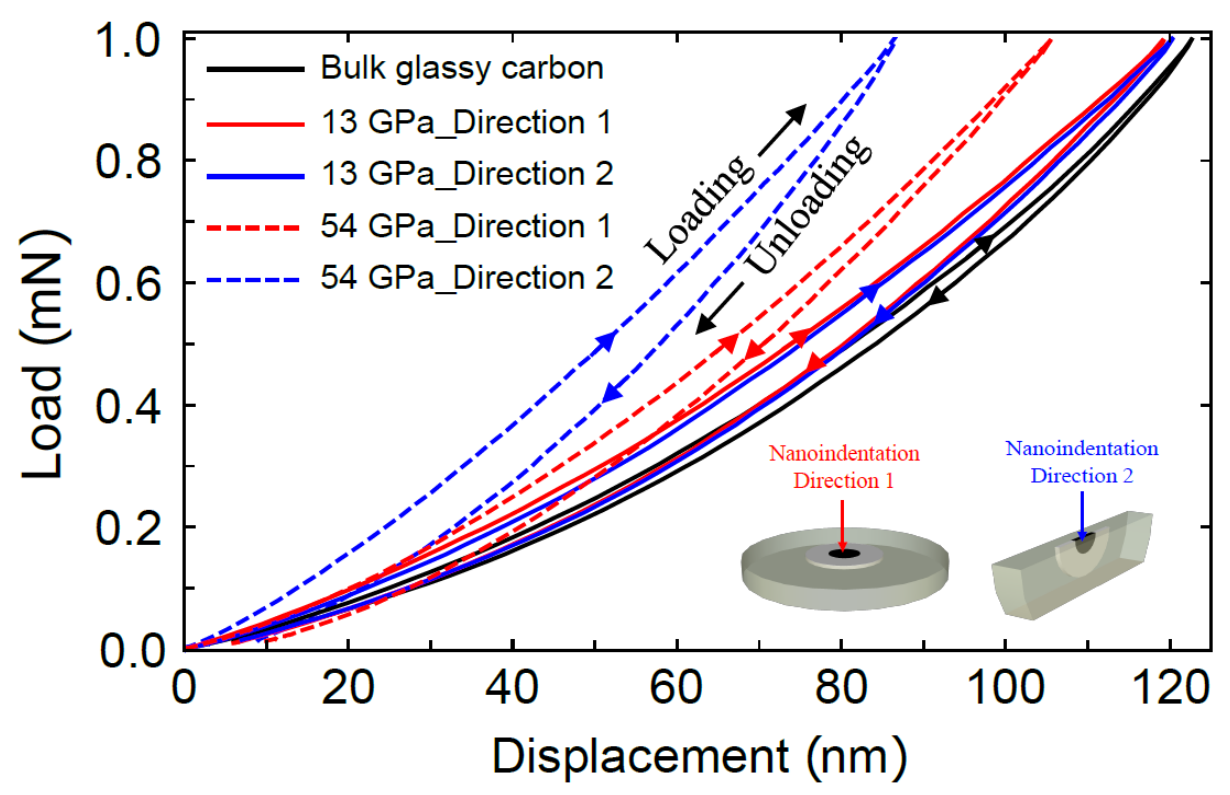

Figure 3: Load-displacement curves of bulk GC sample and samples recovered from $\mathbf{1 3}$ and $54 \mathrm{GPa}$ in the two directions. The loading and unloading curves are labelled on the curve of 54 GPa sample in direction 2.

Next the potential impact of the epoxy substrate at the maximum displacement is considered. Nanoindentation of a layered GC/substrate sample was modelled in the elastic regime using the analytical modelling package Elastica [42]. The simulation used a $10 \mu \mathrm{m}$ film of GC, which is the thinnest among all the samples measured, to maximise the impact of the epoxy. Three different substrates were used, with mechanical properties ranging from very hard (give values), to the epoxy substrate used, to very soft (give values). The calculated result is shown in Fig. 4. At the maximum load, the penetration depths were $~ 76,80$ and $81 \mathrm{~nm}$ for the hard material, epoxy and the soft material respectively. This suggests that the maximum effect of the substrate shows only a difference of $5 \mathrm{~nm}$ on the nanoindentation data. Therefore, the $\sim 20$ $\mathrm{nm}$ difference in the maximum depths on nanoindentation curves in direction 1 and direction 2 of the $54 \mathrm{GPa}$ sample (Fig. 3) can be attributed to a difference in the microstructure. 


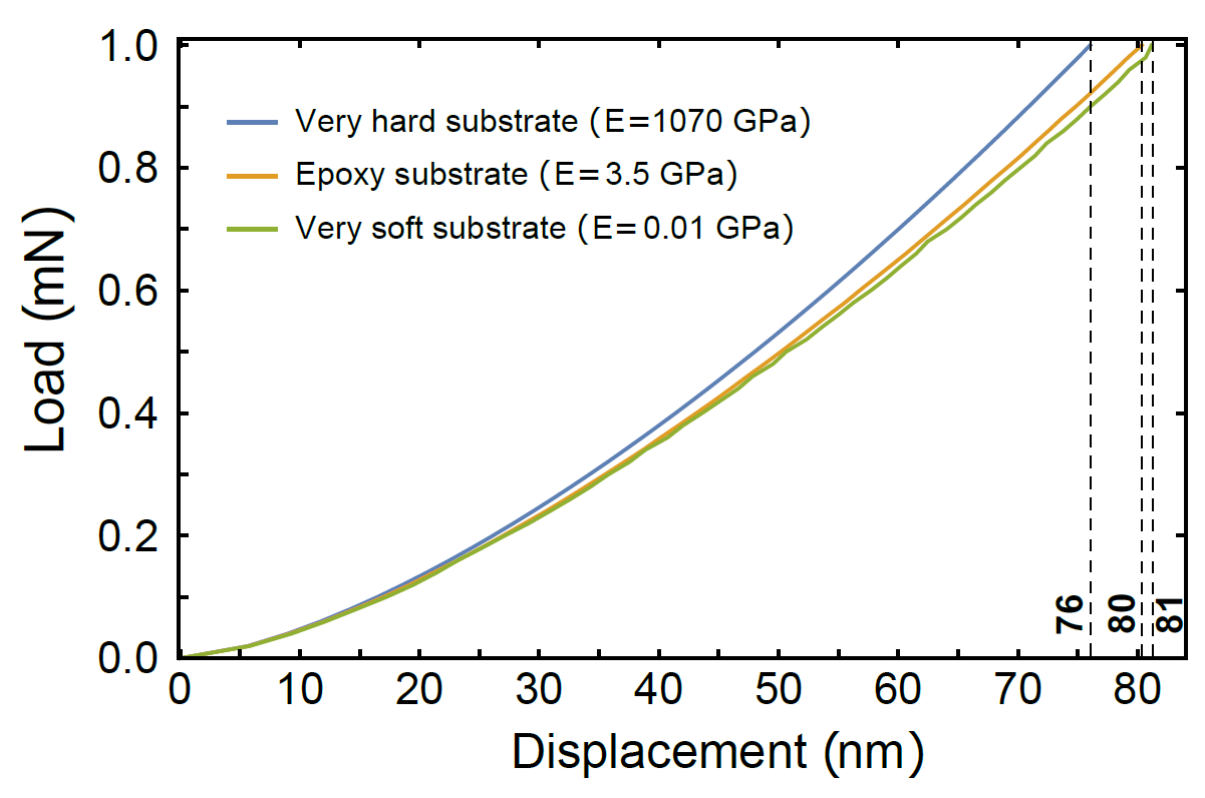

Figure 4: Elastica simulated nanoindentation curves on a $10 \mu \mathrm{m}$ film of glassy carbon on three different substrates: a very hard substrate, the epoxy substrate and a very soft substrate. The maximum penetration depths are labelled.

The elasticity index $E$ is calculated from the experimental nanoindentation curves with the maximum load of $1 \mathrm{mN}$ for all recovered samples. $E$ is plotted as a function of pressure for both directions in Fig. 5. Elasticity indexes in direction 1 are shown in red (triangle markers) and those in direction 2 are shown in blue (square markers). The errors reflect the spread in the data from at least 10 indents for each sample. We measured the elasticity index of bulk GC as $\sim 0.93 \pm 0.03$, which is within the error bar of both direction for zero pressure. 


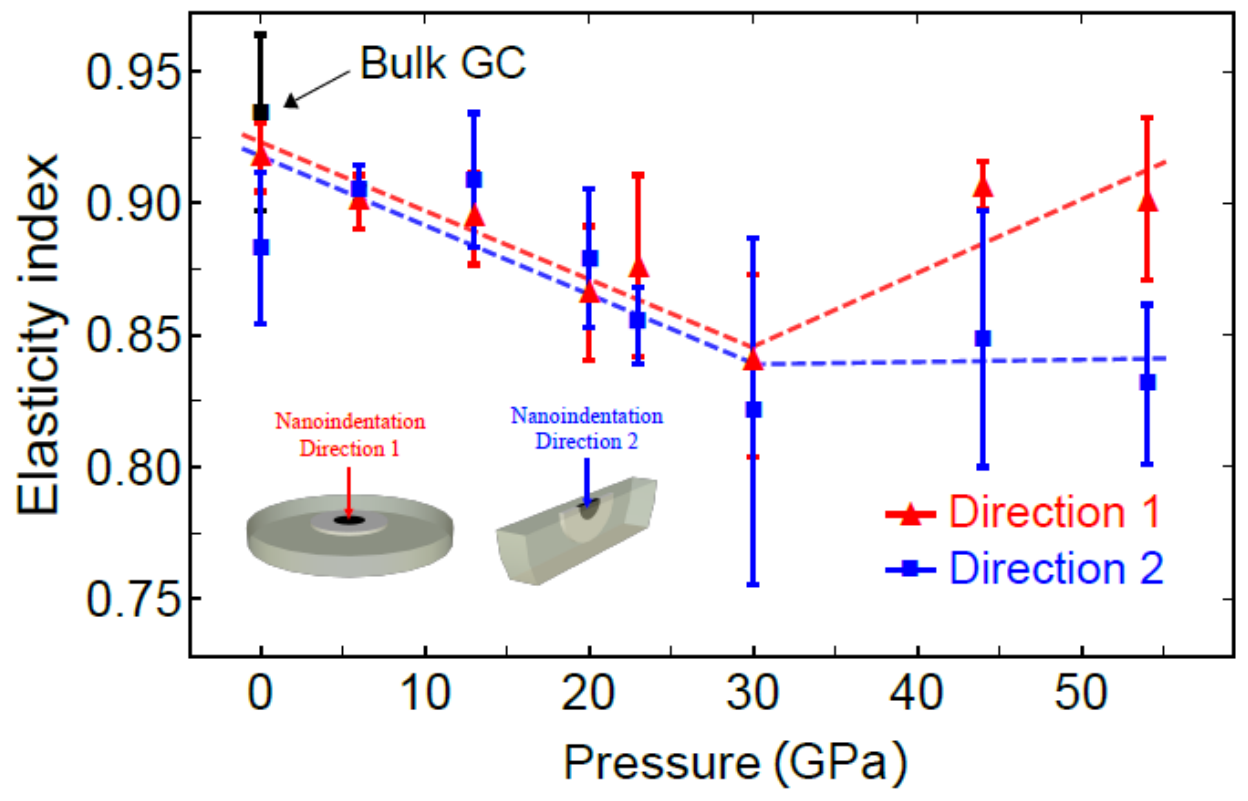

Figure 5: The elasticity indexes $(E)$ of b ulk GC and GC samples recovered from different pressures in two directions. The indexes are averaged from at least 10 indents and the error bars reflect the spread of data. A least squares regression method was used to fit two linear lines both before and after $30 \mathrm{GPa}$.

Figure 5 shows that $E$ decreases between 10 and $30 \mathrm{GPa}$ for both directions. However, for pressures above $\sim 30 \mathrm{GPa}$ it increases for direction 1 while remains constant for direction 2 . Thus after compression above $\sim 30 \mathrm{GPa}$, it is clear that GC is no longer mechanically isotropic. This is in keep with previous TEM observations that the isotropic microstructure of GC breaks down after compression $[15,26]$. However, the change in the mechanical behaviour observed here occurs at lower pressures than expected, based on our previous work which found little difference in the microstructure of GC recovered from compressions up to $40 \mathrm{GPa}$ [15]. The results presented in this current study instead suggest a gradual loss of elasticity at lower pressures. Interestingly, difference observed in the elasticity index between the two directions above $30 \mathrm{GPa}$ was not evident in the residual indent images (Fig. 2), as there was no significant difference in the residual depths (the end of unloading curves in Fig. 3). It is explained from 
the nanoindentation curves according to the definition of elasticity index in equation 1 . As shown in Fig. 3, in direction 1 the curve of $54 \mathrm{GPa}$ has a similar $U_{h}$ value with that of bulk GC, such that $U_{h}$ and $U_{\text {loading }}$ are both decreasing and thus have similar $D$ values. As a result, they also have similar $E$ values. However in direction 2, the curve of $54 \mathrm{GPa}$ is much larger than that of bulk GC, where $U_{h}$ almost the same as but $U_{\text {loading, }}$, is smaller than that of bulk GC, which gives a larger $D$ value. As a result, $E$ of $54 \mathrm{GPa}$ sample is smaller than that of bulk GC in direction 2.

Structures generated using MD simulations can explain the anisotropic mechanical response of GC after subjected to high pressures. The uncompressed GC structure ('Original GC') is shown in Fig. 6(a)-left, which contains 32,768 atoms and has a density of $1.5 \mathrm{~g} / \mathrm{cm}^{3}$. Atoms are coloured according to hybridization, with blue, green and red corresponding to $s p^{3}, s p^{2}$ and $s p$ bonded atoms, respectively. Using this structure to start, a series of compression simulations were performed to $10,25,35$ and $45 \mathrm{GPa}$. After compression to the target pressure, each structure was decompressed to ambient pressure, mimicking the experimental setup. The evolution of the structure during the compression-decompression simulation is shown in Fig. 6(a), where cross-sectional images of the structures at and recovered from $35 \mathrm{GPa}$ are shown as an example (middle and right).

The recovered structure [Fig. 6(a) right] has a density of $1.94 \mathrm{~g} / \mathrm{cm}^{3}$ and it is evident that following compression, graphitic planes showed a preferred orientation perpendicular to the compression axis. It must also be noted that during DAC compression, there is negligible increase in the diameter of the gasket hole as the increasing pressure. Thus the compression of the sample is predominantly along the DAC compression axis and the volume reduction is effectively 1 dimensional, which is comparable with these simulations. 
The elastic constant tensor were computed for the original GC structure and the recovered structures after compressions, then the Young's modulus is calculated from the elastic constant tensor using the Hill formulae [43] in the simulated compression axis (direction 1), as shown in Fig. 6(b). The Young's modulus of the uncompressed GC structure was calculated to be $\sim 42$ $\mathrm{GPa}$, which is in good agreement with the experimental value of $48 \pm 3 \mathrm{GPa}$ [23]. Figure 6(b) shows that the Young's modulus starts to decrease at $10 \mathrm{GPa}$ and reaches the lowest value at $25 \mathrm{GPa}$ before increasing again. This trend is consistent with the experimentally-measured elasticity index along direction 1. 


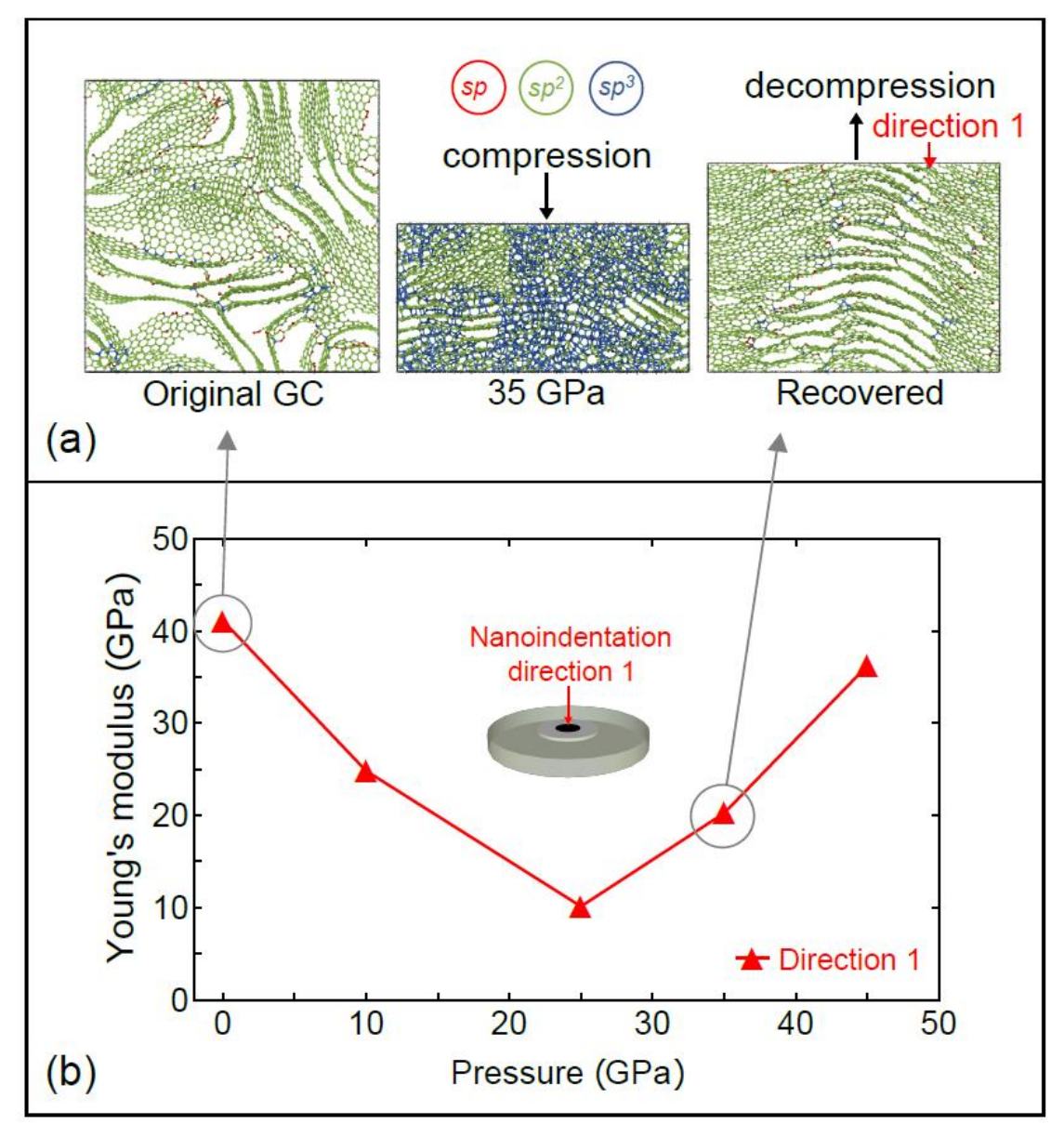

Figure 6: (a) Cross-sectional images showing the atomistic models of GC during key stages of the simulated compression-decompression experiment. Left: original GC at ambient pressure. Middle: GC structure compressed at 35 GPa. Right: recovered structure after decompression from 35 GPa to ambient. Atoms are coloured by coordination number: blue, green and red correspond to $s p^{3}, s p^{2}$ and $s p$ bonded atoms, respectively. The red arrow indicates the axis of compression-decompression and is referred to as 'direction 1' in the nanoindentation experiments. (b) Young's modulus computed along direction 1 on the original GC structure and on the recovered structures after decompression from 10, 25, 35 and 45 GPa to ambient.

It was suggested by Iwashita et al. in 2001 [4] that the strong hysteresis behaviour in the loaddisplacement nanoindentation curves of bulk GC may be understood in terms of reversible slip 
of carbon plane layers, which includes plastically deformation. We refer to this concept as "reverse plasticity" in the following discussion. It is related with the molecular structure and is more complicated than plastically-deformed brittle materials due to particular stress state, like glass. However, key details, namely what aspects of the structure of GC could support reverse plasticity, remained unclear at that time. We now discuss this gap in light of the current results.

Reverse plasticity describes the mechanical deformation of a structure containing a region or 'defect' with a low elastic-plastic transition (yield point) surrounded by material with a higher yield point, as the reversible plastic response model [44]. Thus on loading, the low-yield region may plastically deform before the high-yield surrounding material. On unloading, the stored elastic energy in the elastically-compressed surrounding material will reverse the forces on the defective plastically-deformed region, causing it to again yield and return to something closely resembling its original form. The structure of uncompressed GC is thought to consist of tangled sheet-like arrangements containing open voids and graphene-like structures $[1,2,15]$. Thus in terms of the mechanical response, these open volumes could act as the 'defects' in the reversible plasticity model under small-load nanoindentation. The region containing open volumes would have a lower yield point, which enables the material to plastically deform. The stored elastic energy during loading in the surrounding graphene-sheets could recreate voids on unloading, giving rise to the hysteresis/reverse plasticity observed.

By compressing GC to below $\sim 30 \mathrm{GPa}$, no permanent change in microstructure was observed but we observed a slight increase in the density [15]. This is presumably because the layer spacing between the graphene sheets is reduced and those open volumes or voids, which are critical for the material to 'bounce back' in all directions, are compromised. Such that GC becomes less elastic in both directions. But by compressing GC to above $\sim 30 \mathrm{GPa}$, where $s p^{3}$ 
bonds were reported to form rapidly and the density of the recovered structures also increased rapidly in our previous study [15], the microstructure is permanently changed after decompression. The entangled graphene sheets are oriented [Fig. 6(a)-right] and the layered microstructure of open volumes can still be compressed. These layered volumes will act as new lower-yielding regions that support the reversible plastic response in direction 1, which explains that both the measured elastic index and the computed Young's modulus returned to close to that of the bulk GC in the direction of the compression axis ('direction 1' in Fig. 1 and Fig. 6). However in the direction perpendicular to the compression axis ('direction 2' in Fig. 1), the layer spacing between the sheets cannot be reduced as easily as those in the compression axis, leaving much less lower-yielding regions along this direction.

It needs to be finally clarified that the maximum nanoindentation hydrostatic stress induced by the nanoindentation tip itself is calculated to be $\sim 1 \mathrm{GPa}$ at maximum load (load/cross-sectional contact area at maximum load) for all samples, which is not significant when compared with the pressures used to compress GC in the DAC. Thus, DAC compression is considered as the reason for structural change in this study.

Nanoindentation, which is used as the technique to measure the mechanical response, is sensitive enough to test the structural changes which was not clearly showed in previous TEM studies. Indeed this is not without precedent. A study of on two forms of pure amorphous silicon found they could be differentiated readily using nanoindentation but not using TEM $[45,46]$.

\section{Conclusion}

In conclusion, we have presented an experimental and computational study of the compressed GC, which significantly deepened our understanding of its mechanical properties. We show 
that the mechanical response of GC is less elastic after compression to as low as $6 \mathrm{GPa}$. After compression to $30 \mathrm{GPa}$ there is a permanent microstructural change and the material is no longer mechanically isotropic. Using atomistic simulations we show that the loss of isotropy is due to an irreversible alignment of the graphene-like layers. 


\section{Acknowledgements}

The authors would like to acknowledge the Australian Research Council (ARC) for funding under the ARC Discovery Project scheme (DP190101438, DP170102087 and DP140102331), and M. V. Swain for useful discussions. 


\section{References}

[1] G. M. Jekins, K. Kawamura, Nature, 175 (1971). doi: 10.1038/231175a0

[2] P. J. F. Harris, Philosophical Magazine 84, 3159 (2004). doi: $10.1080 / 14786430410001720363$

[3] M. V. Swain and J. S. Field, Philosophical Magazine A 74, 1085 (1996). doi: $10.1080 / 01418619608239709$

[4] N. Iwashita, M. V. Swain, J. S. Field, N. Ohta, S. Bitoh, Carbon, 1525 (2001). doi: $10.1016 / \mathrm{S} 0008-6223(00) 00272-4$

[5] M. Li, J. Guo and B. Xu, Applied Physics Letters 102, 121904 (2013). doi: $10.1063 / 1.4798556$

[6] F. C. Cowlard, J. C. Lewis, Journal of Materials Science 2, 507 (1967). doi: 10.1007/BF00752216

[7] Z. Zhang, R. Brydson, Z. Aslam, S. Reddy, A. Brown, A. Westwood, B. Rand, Carbon, 5049 (2011). doi: 10.1016/j.carbon.2011.07.023

[8] T. B. Shiell, D. G. McCulloch, J. E. Bradby, B. Haberl, R. Boehler and D. R. McKenzie, Scientific Reports 6, 37232 (2016). doi: 10.1038/srep37232

[9] S. Wong, T. B. Shiell, B. A. Cook, J. E. Bradby, D. R. McKenzie, D.G. McCulloch, Carbon 142, 475 (2019)

[10] T. Sekine, M. Akaishi, N. Setaka and K. I. Kondo, Journal of Materials Science 22(10), 3615 (1987). doi: 10.1007/BF01161469

[11] K. Higashi and A. Onodera, Physica 139 \&140B, 813 (1986).

[12] H. Sumiya and T. Irifune, Journal of Materials Research 22(8), 2345 (2007). doi: 10.1557/jmr.2007.0295

[13] Z. Zeng, L. Yang, Q. Zeng, H. Lou, H. Sheng, J. Wen, D. J. Miller, Y. Meng, W. Yang, 
W. L. Mao and H. K. Mao, Nature Communications 8(1), 322 (2017). doi: $10.1038 / \mathrm{s} 41467-017-00395-\mathrm{w}$

[14] M. Hu, J. He, Z. Zhao, T. A. Strobel, W. Hu, D. Yu, H. Sun, L. Liu, Z. Li, M. Ma, Y. Kono, J. Shu, H. Mao, Y. Fei, G. Shen, Y. Wang, S. J. Juhl, J. Y. Huang, Z. Liu, B. Xu and Y. Tian, Science Advances 3, e1603213 (2017). doi:10.1126/sciadv.1603213

[15] T. B. Shiell, D. G. McCulloch, D. R. McKenzie, M. R. Field, B. Haberl, R. Boehler, B. A. Cook, C. de Tomas, I. Suarez-Martines, N. A. Marks, and J. E. Bradby, Physical Review Letters 120, 215701 (2018). doi: 10.1103/PhysRevLett.120.215701

[16] Y. Lin, L. Zhang, H. Mao, P. Chow, Y. Xiao, M. Baldini, J. Shu and W. L. Mao, Physical Review Letters 107, 175504 (2011). doi: 10.1103/PhysRevLett.107.175504

[17] Z, Zhao, E. F. Wang, H. Yan, Y. Kono, B. Wen, L. Bai, F. Shi, J. Zhang, C. KenneyBenson, C. Park, Y. Wang and G. Shen, Nature Communications 6, 6212 (2015). doi: $10.1038 /$ ncomms 7212

[18] M. Yao, X. Fan, W. Zhang, Y. Bao, R. Liu, B. Sundqvist and B. Liu, Applied Physics Letters 111, 101901 (2017). doi: 10.1063/1.4996278

[19] M. Yao, J. Xiao, X. Fan, R. Liu, and B. Liu, Applied Physics Letters 104, 021916 (2014). doi: 10.1063/1.4861929

[20] J .S. Field and M. V. Swain, Carbon 34, 1357 (1996)

[21] W. C. Oliver and G. M. Pharr, Journal of Materials Research 19, 3 (2004). doi: 10.1557/jmr.2003.19.1.3

[22] S. Basu, A. Moseson and M. W. Barsoum, Journal of Materials Research 21, 2628 (2006). doi: 10.1557/JMR.2006.0324

[23] T. B. Shiell, S. Wong, W. Yang, C. Tanner, B. Haberl, R. G. Elliman, D. R. McKenzie, D. G. McCulloch and J. E. Bradby, Journal of Non-Crystalline Solid 522, 119561 (2019). doi: 10.1016/j.jnoncrysol.2019.119561 
[24] S. Plimpton, Journal of Computational Physics, 117, 1-19 (1995). doi: 10.1006/jcph.1995.1039

[25] N. A. Marks, Physical Review B - Condensed Matter and Materials Physics, 63(3), 1-7 (2001). doi: 10.1103/PhysRevB.63.035401

[26] T. B. Shiell, C. de Tomas, D. G. McCulloch, D. R. McKenzie, A. Basu, I. SuarezMartinez, N. A. Marks, R. Boehler, B. Haberl, and J. E. Bradby, Physical Review B 99, 024114 (2019). doi: 10.1103/PhysRevB.99.024114

[27] C. de Tomas, I. Suarez-Martinez, N. A. Marks, Carbon 109, 681 (2016). doi: 10.1016/j.carbon.2016.08.024

[28] M. Sakai, Acta Metallurgica et Materialia 41, 1751 (1993).

[29] R. Nowak and M. Sakai, Journal of Materials Research 8, 5 (1993).

[30] W. C. Oliver and G. M. Pharr, Journal of Materials Research 7, 1564 (1992). doi: 10.1557/JMR.1992.1564

[31] B. J. Briscoe, L. FIori and E. Pelillo, Journal of Physics D: Applied Physics 31, 2395 (1998). doi: 10.1088/0022-3727/31/19/006

[32] A. C. Fischer-Cripps, Materials Science and Engineering A 385, 74 (2004). doi: 10.1016/j.msea.2004.04.070

[33] M. L. Oyen, Journal of Biomechanic 39, 2699 (2006). doi: 10.1016/j.jbiomech.2005.09.011

[34] R. Boehler, Review of Scientific Instruments 77, 115103 (2016). doi: $10.1063 / 1.2372734$

[35] A. Albiez and R. Schwaiger, MRS Advances, 4(2), 133-138 (2019). doi: $10.1557 / \mathrm{adv} .2018 .648$

[36] X. Zhang, A. Vyatskikh, H. Gao, J. R. Greer and X. Li, Proceedings of the National Academy of Sciences of the United States of America, 116(14), 6665-6672 (2019). 
doi: 10.1073/onas.1817309116

[37] A. Dewaele, M. Torrent, P. Loubeyre and M. MezouaR, Physical Reciew B 78, 104102 (2008). doi: 10.1103/PhysRevB.78.104102

[38] H. K. Mao, J. Xu, and P. M. Bell, Journal of Geophysical Research 91, 4673 (1986)

[39] Y. Akahama and H. Kawamura, Journal of Applied Physics 100, 043516 (2006). doi:10.1063/1.2335683

[40] N. A. Solopova, N. Dubrovinskaia and L. Dubrovinsky, Applied Physics Letters 102, 121909 (2013). doi: $10.1063 / 1.4798660$

[41] R. Saha, W. D. Nix, Acta Materialia 50, 23 (2002)

[42] Elastica, ASMEC GmbH, Germany

[43] C. de Tomas, I. Suarez-Martinez, N.A. Marks, Applied Physics Letters 112, 251907 (2018). doi: $10.1063 / 1.5030136$

[44] N. Iwashita, J. S. Field and M. V. Swain, Philosophical Magazine A 82, 1873 (2002). doi: $10.1080 / 01418610208235699$

[45] B. Haberl, J. E. Bradby, S. Ruffell, J. S. Williams and P. Munroe, Journal of Applied Physics, 100(1) (2006). doi: 10.1063/1.2210767

[46] B. Haberl, A. C. Y. Liu, J. E. Bradby, S. Ruffell, J. S. Williams and P. Munroe, Physical Review B - Condensed Matter and Materials Physics, 79(15), 1-8 (2009). doi: 10.1103/PhysRevB.79.155209 
(a)

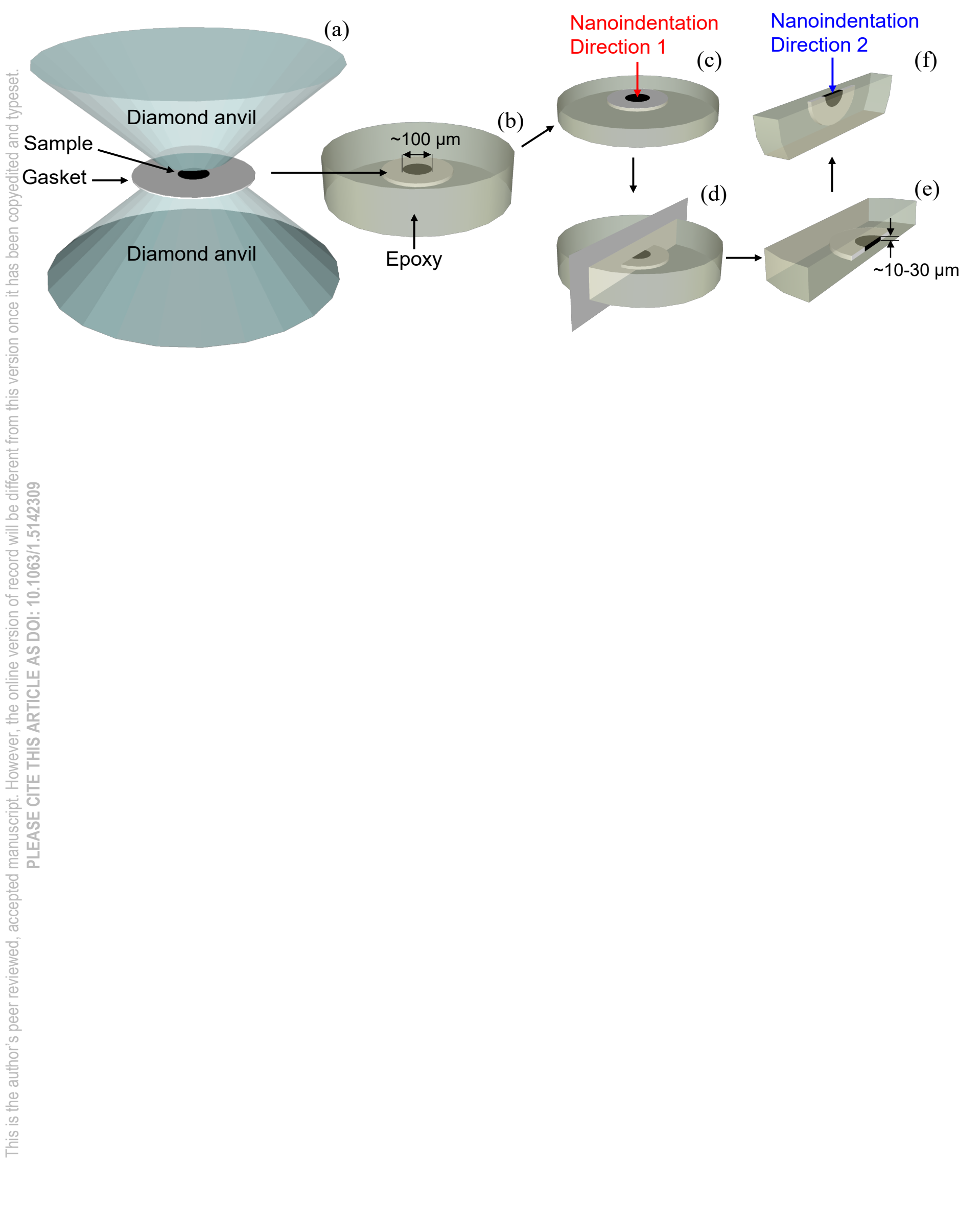




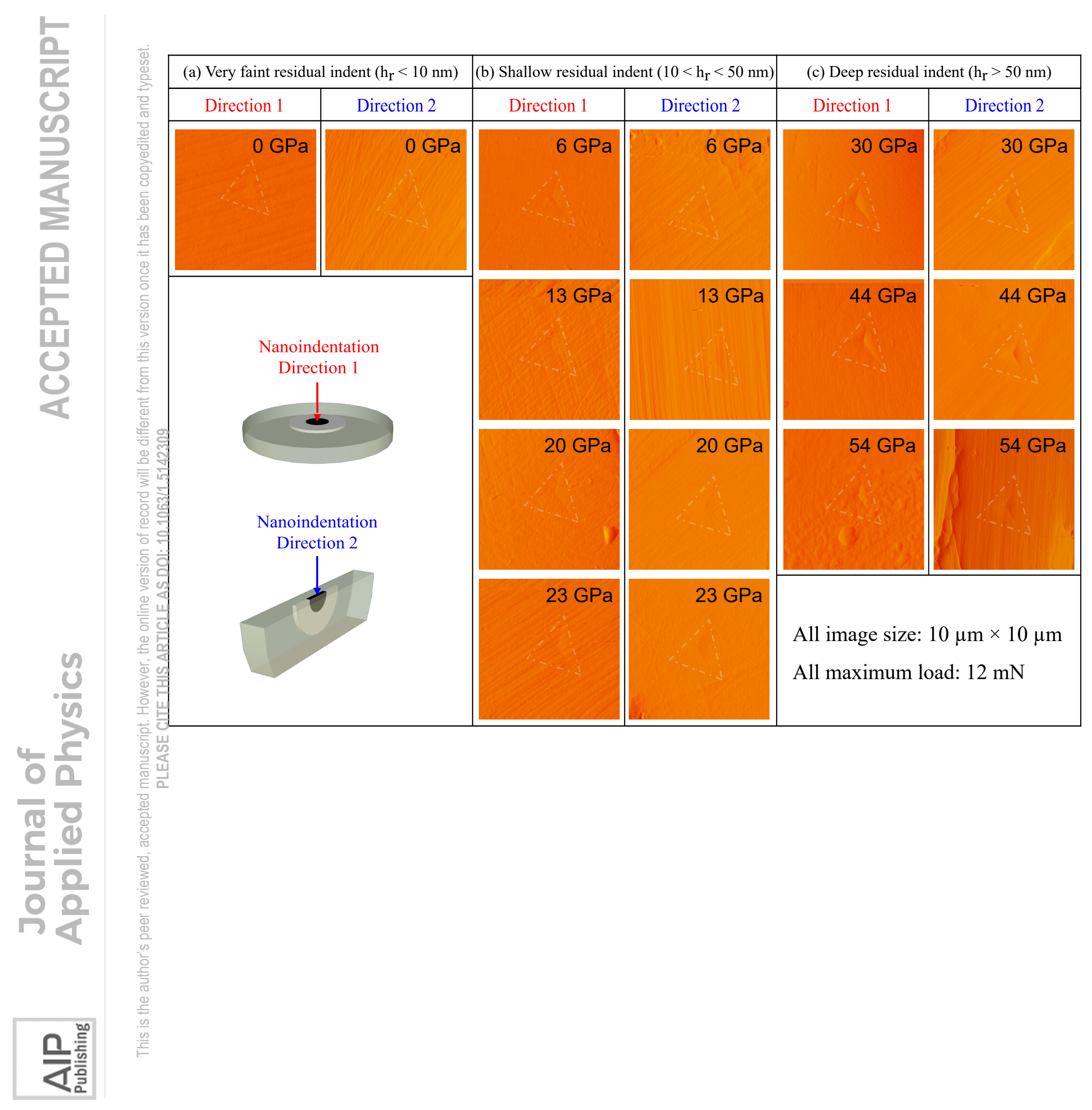




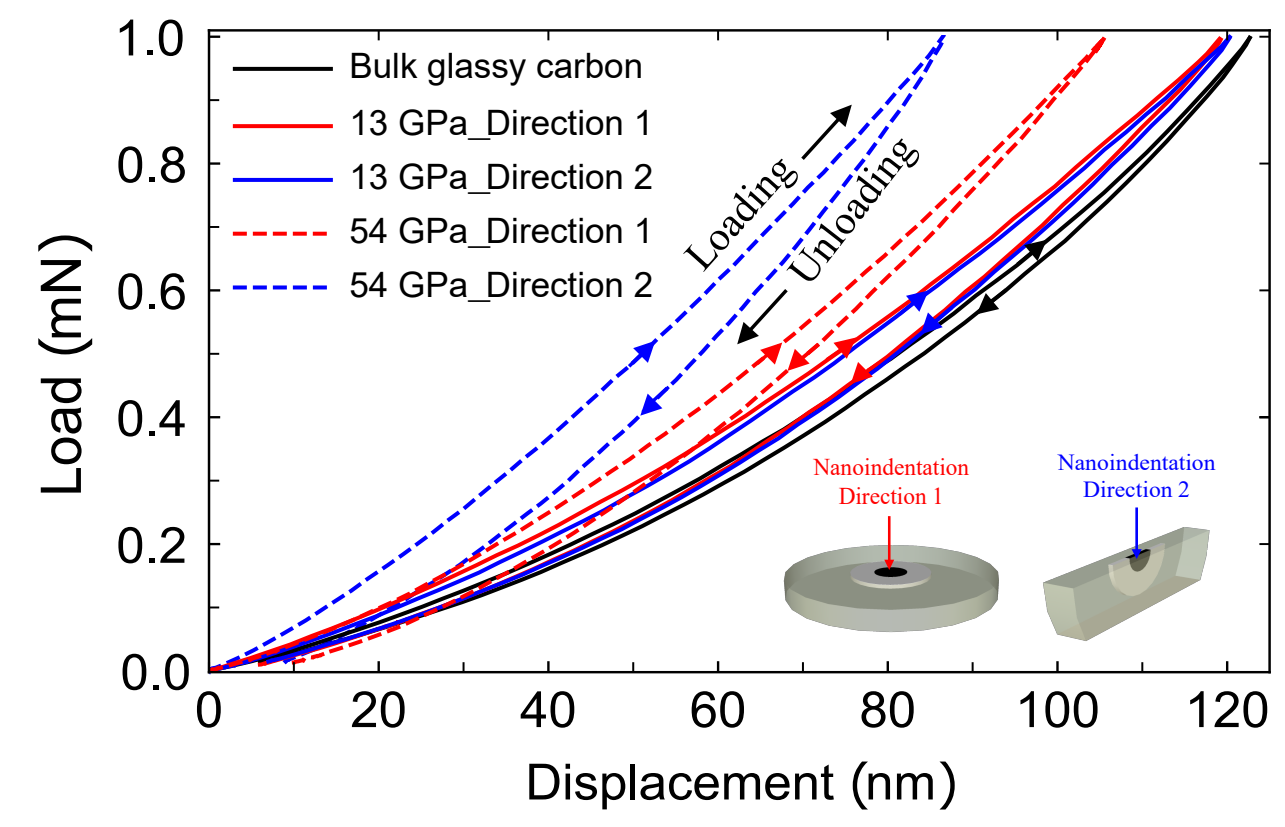




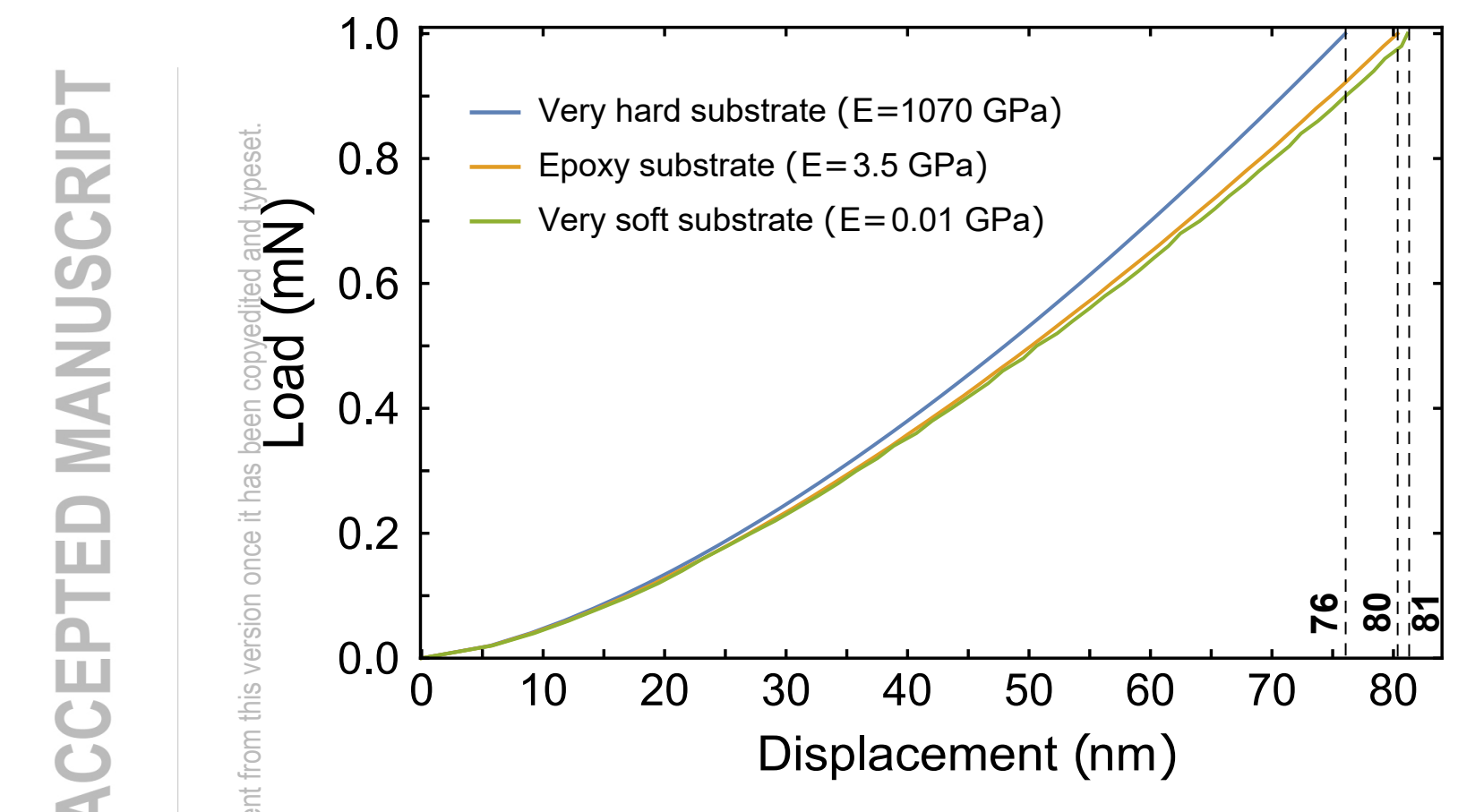




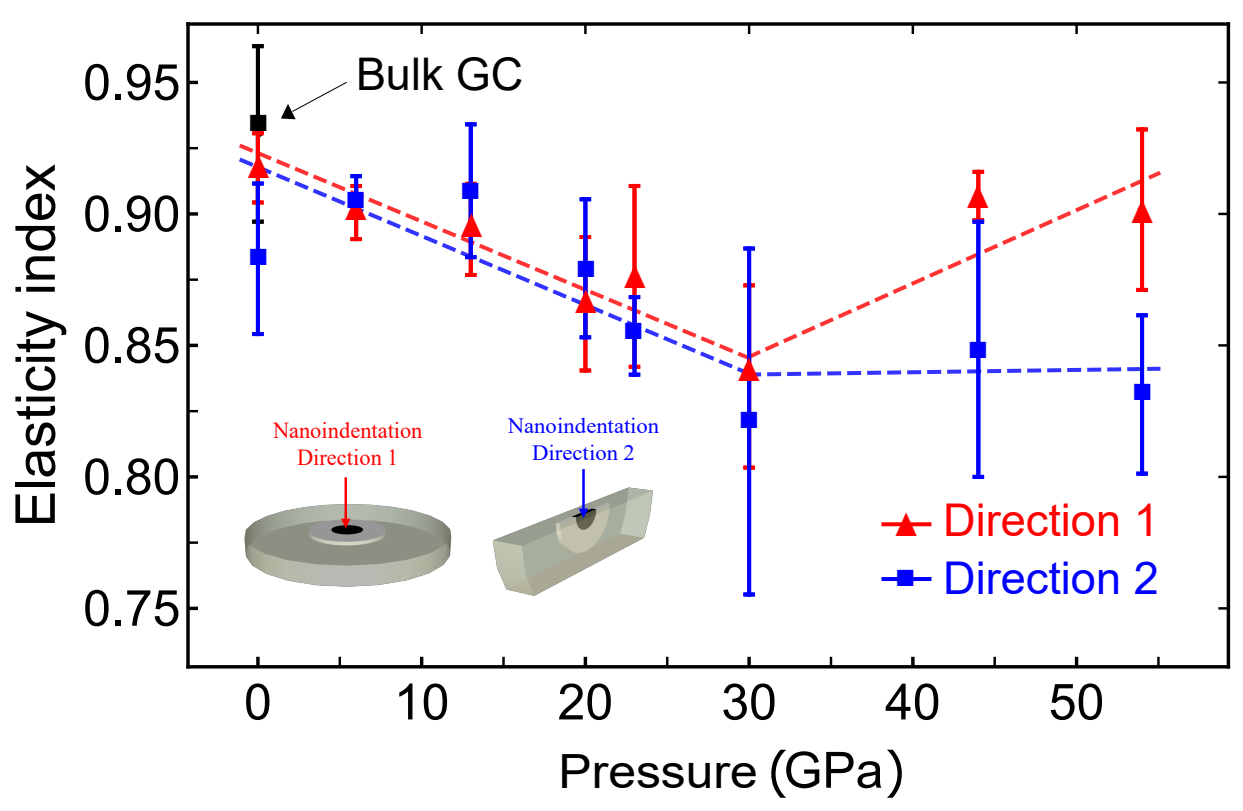



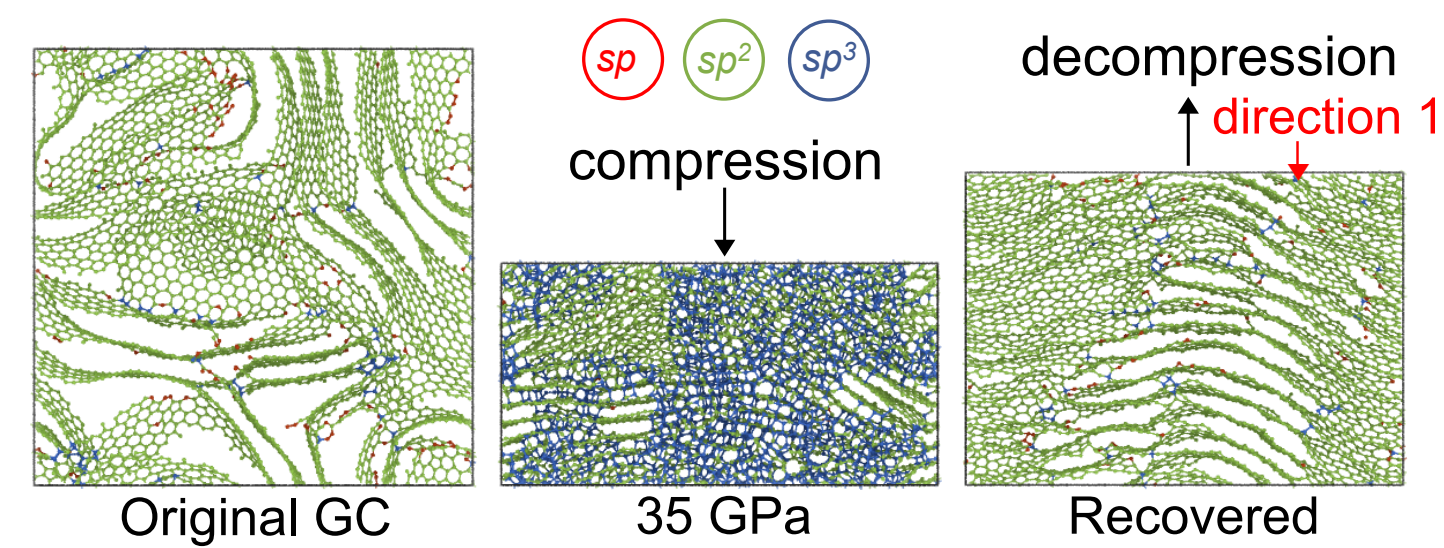

(a)

Original GC

$35 \mathrm{GPa}$

Recovered

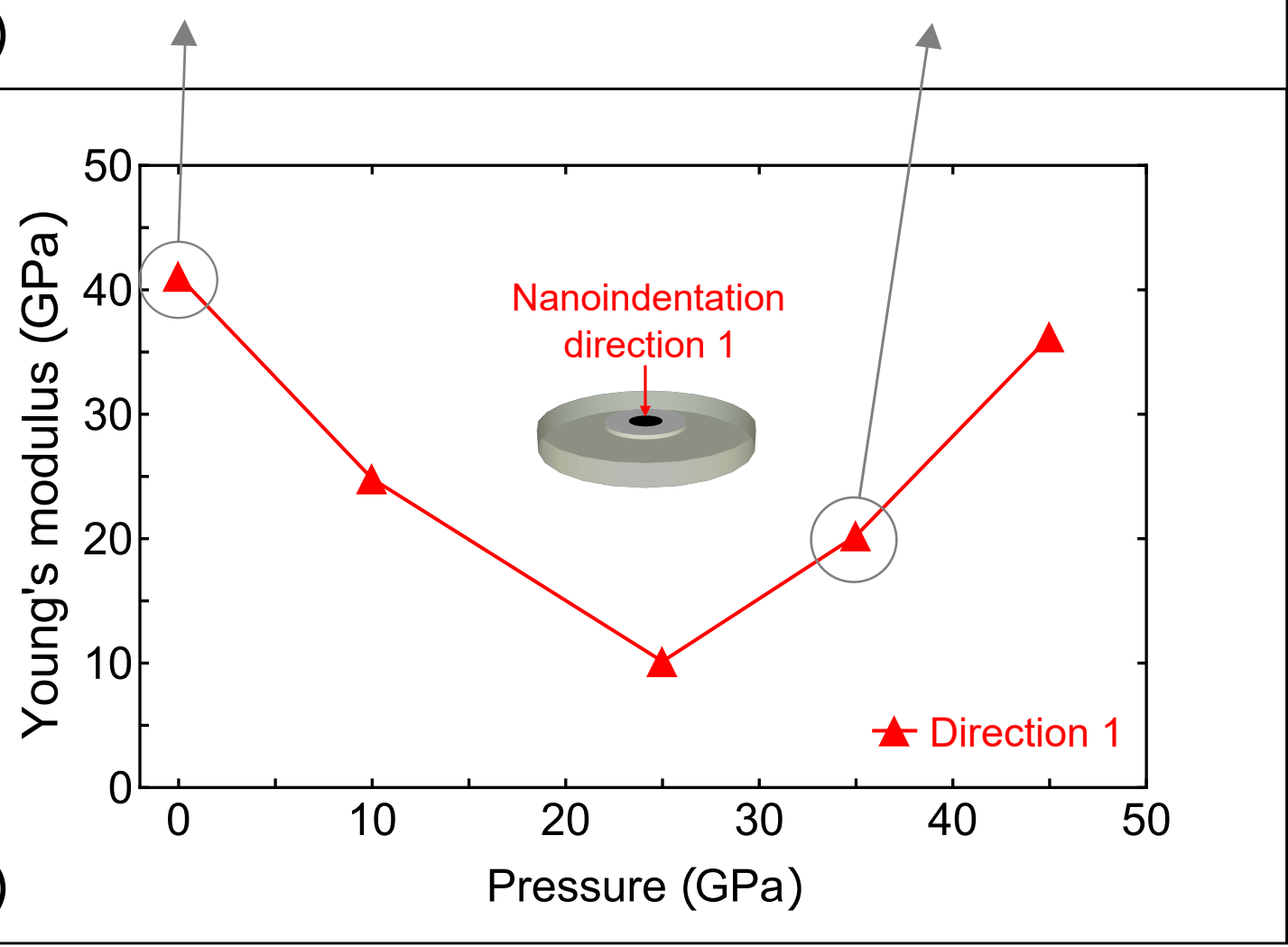

呈产产 